\title{
Level Crossing Rates and MIMO Capacity Fades: Impacts of Spatial/Temporal Channel Correlation
}

\author{
Andrea Giorgetti*, Marco Chiani*, Mansoor Shafi ${ }^{\dagger}$ and Peter J. Smith ${ }^{\ddagger}$ \\ ${ }^{*}$ DEIS University of Bologna, V.le Risorgimento 2, 40136 Bologna, Italy. \\ Email: \{agiorgetti,mchiani\}@deis.unibo.it \\ ${ }^{\dagger}$ Telecom New Zealand, PO Box 293, Wellington, New Zealand. \\ Email: Mansoor.Shafi@telecom.co.nz \\ $\ddagger$ Department of Electrical and Computer Engineering, The University of Canterbury, \\ Private Bag 4800 Christchurch, New Zealand. \\ Email: p.smith@elec.canterbury.ac.nz
}

\begin{abstract}
It is well known that MIMO systems offer the promise of achieving very high spectrum efficiencies (many tens of bit/s/Hz) in a mobile environment. The gains in MIMO capacity are sensitive to the presence of spatial and temporal correlation introduced by the radio environment. In this paper we examine how MIMO capacity is influenced by a number of factors e.g. a) temporal correlation b) various combinations of low/high spatial correlations at either end, c) combined spatial and temporal correlations. In all cases we compare the channel capacity that would be achievable under independent fading. We investigate the behaviour of "capacity fades", examine how often the capacity experiences the fades, develop a method to determine level crossing rates and average fade durations and relate these to antenna numbers.
\end{abstract}

\section{INTRODUCTION}

Since the pioneering work of Winters [1], Telatar [2], Foschini and Gans [3], Multiple Input Multiple Output (MIMO) systems have received considerable attention in recent years as they have the potential to provide quantum leaps in capacity [4]. The gains in MIMO shown in [3] are for independent fading amongst the antenna elements. In order to fully evaluate the impacts of correlation on MIMO capacity one needs to consider the combined effects of all sources of channel degradation pertinent to a MIMO channel, namely angle spread of the arriving multipath signal, Doppler spectrum and the delay power spectrum. In the open literature there appear to be no expressions for this combined spectrum. If, however, the constituent spatial, temporal and spectral correlations are assumed independent, then a combined correlation is simply the product of the constituent correlations. Abdi and Kaveh [5] have developed closed form expressions for spectral-temporal correlation that take into account various parameters of interest such as angle spreads at the base station and user end, array configurations, Doppler spreads etc. They have used a von Mises distribution for the angles of arrival and departure. The wireless standards body $3 \mathrm{GPP}$ has published a standardized set of MIMO propagation models [6] that define key parameters and distributions needed to evaluate the combined spatial/temporal/spectral correlation function. In this paper we

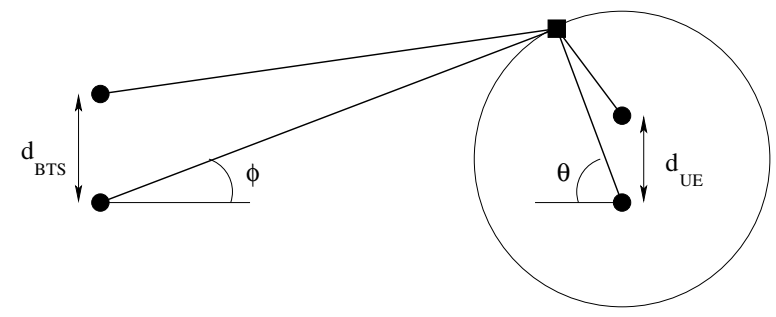

Fig. 1. The one-ring scatterers model for MIMO systems. The number of transmitting and receiving antenna are $n_{T}$ and $n_{R}$, respectively.

have considered the standardized propagation model in [6] and only considered the first two sources of correlation. The consideration of spectral correlation forms part of future work. The time variations of capacity introduced by the temporal correlation lead to the notion of finding periods of time the capacity decreases below a given value. We refer to these periods as "capacity fade". We address the following issues:

- with temporal correlation only, how often does the capacity experience fades and what are their duration?

- are the rates of occurrence of capacity fades and their durations influenced by antenna numbers?

- how do the answers to the above change with the introduction of varying amounts of spatial correlation?

In Section II we describe the MIMO propagation model; capacity fades for spatially/temporally correlated MIMO systems and their approximations are analyzed in Section III; in Section IV we present numerical results in terms of capacity, level crossing rates and average fade duration, and comparisons with approximations.

\section{A GENERALIZED PROPAGATION MODEL FOR THE MIMO CHANNEL}

Let us consider a wireless system with $\left(n_{T}, n_{R}\right)$ antennas as shown in Fig. 1 arranged as linear arrays at either end. The element spacing at the base station and mobile end is denoted by $d_{B T S}$ and $d_{U E}$, respectively. 
Let $h_{m p}$ be the complex channel coefficient between the receiver $m, m=1, \ldots, n_{R}$ and transmitter $p, p=1, \ldots, n_{T}$, then the correlation between two coefficients is given by

$$
\mathbb{E}\left[h_{m_{1} p_{1}} h_{m_{2} p_{2}}^{*}\right]=\mathbb{E}\left[e^{j 2 \pi \frac{d\left(p_{1}, p_{2}\right) \sin \phi}{\lambda}} e^{j 2 \pi \frac{d\left(m_{1}, m_{2}\right) \sin \theta}{\lambda}}\right]
$$

where $\lambda$ is the carrier wavelength, $d\left(m_{1}, m_{2}\right)$ is the distance between the receive antennas $m_{1}$ and $m_{2}$, and $d\left(p_{1}, p_{2}\right)$ is the distance between transmit antennas $p_{1}$ and $p_{2}$. The expectations are taken with respect to the random position of scatterers, i.e. with respect to the random variables $\phi$ and $\theta$. In this paper we assume the angles of departure $\phi$ follow a Laplacian distribution, and a uniform distribution for arrival angles $\theta[6]$.

As far as the channel model is concerned, a MIMO channel is commonly characterized by a $n_{R} \times n_{T}$ channel matrix $\mathbf{H}$ with elements $h_{i j}$. In a rich scattered frequency non-selective environment the elements of $\mathbf{H}$ are complex Gaussian random variables with unit energy. By stacking up the columns of $\mathbf{H}$, we can construct the channel vector $\mathbf{h}$ for this systems as

$$
\mathbf{h}=\left[h_{1,1} \ldots h_{n_{R}, 1} \ldots h_{1, n_{T}} \ldots h_{n_{R}, n_{T}}\right]^{T}
$$

where ()$^{T}$ denotes matrix transpose. Then the channel (spatial) correlation matrix is defined as

$$
\mathbf{R}=\mathbb{E}\left[\mathbf{h h}^{\dagger}\right]
$$

where ()$^{\dagger}$ is the transpose conjugate operator. Now, $\mathbf{R}$ can be found given the statistical distributions of $\phi$ and $\theta$ and by using (1) and (2) in (3). For the purpose of simulation, given $\mathbf{R}$ the correlated channel coefficients $\mathbf{h}_{\text {corr }}$ are simply given by

$$
\mathbf{h}_{\text {corr }}=\mathbf{R}^{1 / 2} \mathbf{h}_{\text {uncorr }}
$$

where $\mathbf{R}^{1 / 2}$ is the matrix square root of $\mathbf{R}$ and $\mathbf{h}_{\text {uncorr }}$ is a vector of uncorrelated channel coefficients.

Usually the channel correlations are separable or at least approximately so. For example in our model, the expectation in (1) is given by a product of correlations between antenna elements at the base station and mobile station end respectively $\rho_{p_{1}, p_{2}}^{B T S} \rho_{m_{1}, m_{2}}^{U E}[5]-[7]$.

Let now us define the correlation matrices at the base station end and mobile end as $\mathbf{R}_{B T S}=\left[\rho_{p, q}^{B T S}\right]_{n_{T} \times n_{T}}$ and $\mathbf{R}_{U E}=$ $\left[\rho_{p, q}^{U E}\right]_{n_{R} \times n_{R}}$.

Then it can be shown that under some conditions it is possible to write [5]-[7]:

$$
\mathbf{R}=\mathbf{R}_{B T S} \otimes \mathbf{R}_{U E}
$$

where $\otimes$ is the Kronecker product. This is true in our model and allows us to independently vary correlation at either end and examine the consequential impact on capacity.

Since we want to take into account not only spatial but also temporal correlation, we assume that the time variations of the channel coefficients in $\mathbf{H}$ are governed by the well known Jakes fading process [8] (see also Section IV).

In conclusion we can consider both time and space correlation and, by varying the maximum Doppler frequency $f_{D}$, element spacings and mean angles of arrival/departure, various scenarios of high and low correlation can be simulated.

\section{MIMO CAPACITY FOR CORRELATED CHANNELS}

We consider a single user $n_{T} \rightarrow n_{R}$ MIMO system operating in a correlated fading environment discussed in section II. The received signal, $\mathbf{r}$, is given by

$$
\mathbf{r}=\mathbf{H} \mathbf{s}+\mathbf{n}
$$

where $\mathbf{r}$, is the $n_{R} \times 1$ received signal, $\mathbf{s}$ is the $n_{T} \times 1$ transmitted signal and $\mathbf{n}$ is an $n_{R} \times 1$ vector of iid AWGN terms normalized so that the elements have unit magnitude variance. The total power of $\mathbf{s}$ is constrained to $\mathbf{P} . \mathbf{H}$ is the $n_{R} \times n_{T}$ channel matrix. Assuming equal power uncorrelated sources (optimum for the case when the transmitter does not know the channel) the capacity is given by [3]

$$
C=\log _{2} \operatorname{det}\left(\mathbf{I}_{n_{R}}+\frac{P}{n_{T}} \mathbf{H} \mathbf{H}^{\dagger}\right) \quad \mathrm{bit} / \mathrm{s} / \mathrm{Hz}
$$

Note that in all this work we assume the "quasi-static" case [3] where the channel varies randomly from burst to burst. Within a burst the channel is assumed fixed and it is also assumed that sufficient bits are transmitted for the standard infinite time horizon of information theory to be meaningful.

The capacity formula is now extremely well-known and the simple process of replacing $\mathbf{H}$ by a sequence of spatiotemporally channel matrices $\mathbf{H}_{n}$ for $n=1,2, \ldots$ results in a temporal sequence of capacity values. The modeling of the channel matrices is given in section II.

It is now reasonably well known that for independent fading the capacity of MIMO systems is approximately Gaussian for small numbers of antennas and converges to the Gaussian distribution as $n_{R} \rightarrow \infty, n_{T} \rightarrow \infty$ and the ratio $n_{R} / n_{T}$ tends to a constant [9], [10]. It is sensible therefore to investigate whether the temporally (and spatially) correlated sequence of capacity values might also be well approximated by a Gaussian sequence. Whether the Gaussian approximation is accurate for correlated fading scenarios is less well-known. Hence in Fig. 6 we compare the complementary cumulative distribution function (ccdf) of the data with a Gaussian ccdf with the same mean and variance. The correlation scenarios used are described in Section IV and the parameters given in Table I. The agreement is excellent and we proceed to investigate whether the temporal behaviour can also be approximated using Gaussian process results.

In order to investigate the temporal behaviour of the capacity we focus on level crossing rates across a level $C_{T}$ (denoted $\operatorname{LCR}\left(C_{T}\right)$ ), and average fade durations (periods of time spent with $C<C_{T}$ denoted $A F D\left(C_{T}\right)$ ). For any discrete time Gaussian model the LCR is trivial and can be calculated as 
below. Let $\rho_{c}$ be the correlation between successive capacity values, $\mu$ be the mean capacity and $\sigma^{2}$ the capacity variance. Defining the standardized capacity values as $\tilde{C}=(C-\mu) / \sigma$ we can relate $\tilde{C}_{n}$ to $\tilde{C}_{n-1}$ as below.

$$
\tilde{C}_{n}=\rho_{c} \tilde{C}_{n-1}+\sqrt{1-\rho_{c}^{2}} U_{n}
$$

where $U_{n}$ is an independent normal variable, i.e. $U_{n} \sim$ $\mathcal{N}(0,1)$. Now an upcrossing across a level defined by $C=C_{T}$ is defined by the pair of events: $C_{n-1}<C_{T}$ and $C_{n}>C_{T}$. In terms of standardized values an upcrossing is also equivalent to the pair of events: $\tilde{C}_{n-1}<\tilde{C_{T}}$ and $\tilde{C}_{n}>\tilde{C_{T}}$ where $\tilde{C_{T}}=$ $\left(C_{T}-\mu\right) / \sigma$. Hence we have

$$
\begin{aligned}
\operatorname{LCR}\left(\tilde{C_{T}}\right) & =\mathbb{P}\left(\tilde{C}_{n-1}<\tilde{C_{T}}, \tilde{C}_{n}>\tilde{C_{T}}\right) \\
& =\mathbb{P}\left(\tilde{C}_{n-1}<\tilde{C_{T}}, U_{n}>\frac{\tilde{C_{T}}-\rho_{c} \tilde{C}_{n-1}}{\sqrt{1-\rho_{c}^{2}}}\right) \\
& =F\left(\tilde{C_{T}}\right)-\int_{-\infty}^{\tilde{C_{T}}} f(x) F\left(\frac{\tilde{C_{T}}-\rho_{c} x}{\sqrt{1-\rho_{c}^{2}}}\right) d x
\end{aligned}
$$

where $f(x)$ and $F(x)$ are the density and distribution function respectively of the standard Gaussian distribution. Note that this only requires the correlation parameter $\rho_{c}$. At present this parameter is simply estimated from the data as an analytical solution appears complicated. More generally, of course, we could attempt to fit a particular model, perhaps a Gaussian ARMA(p,q) model, to the capacity sequence. This is beyond the scope of this paper.

Another approach to the level crossing problem is to use Rice's Formula for the continuous time case. By using quite standard results from stochastic process theory it can be shown that, if $\tilde{C}(t)$ is a (standardized) continuous time Gaussian process with autocorrelation function $\rho_{c}(\tau)$, then the upcrossing rate across the level $\tilde{C}_{T}$ is given by [8], [11]

$$
\frac{\left(-\ddot{\rho}_{c}(0)\right)^{1 / 2}}{2 \pi} \exp \left(-\frac{{\tilde{C_{T}}}^{2}}{2}\right) .
$$

Some further calculations show that $-\ddot{\rho}_{c}(0)=$ $\operatorname{var}(d \tilde{C}(t) / d t)$, hence relating the constants to variances of the process rather than the autocorrelation function (ACF). At this stage we do not have any analytic results for $\rho_{c}(\tau)$ or $\operatorname{var}(d \tilde{C}(t) / d t)$ but the form of this result can be easily checked by evaluating $\ddot{\rho}_{c}(0)$ by the data, or by a simple scaling of the $\exp \left(-{\tilde{C_{T}}}^{2} / 2\right)$ curve.

However the LCR is calculated, it can be related to the AFD using the result [8]

$$
A F D\left(\tilde{C_{T}}\right)=\frac{F\left(\tilde{C_{T}}\right)}{\operatorname{LCR}\left(\tilde{C_{T}}\right)}
$$

In section IV we investigate how closely these simple Gaussian process approximations match our discrete time simulations.

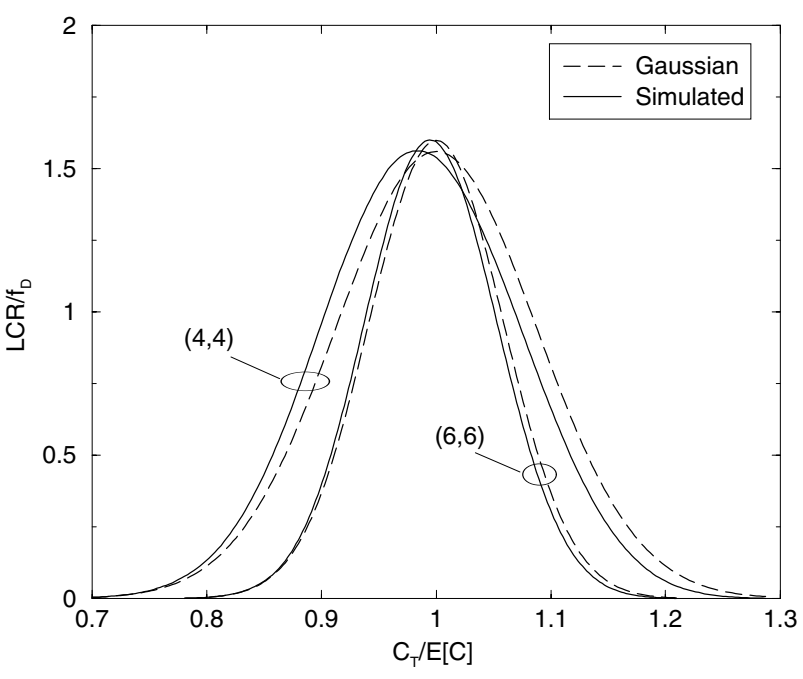

Fig. 2. LCR of the capacity without spatial correlation for $P=20 \mathrm{~dB}$ : comparison between Gaussian process approximation and simulation.

\section{RESUlTS}

The simulation of MIMO channels with temporal correlation only requires the generation of multiple complex waveforms satisfying the following conditions. First, the real and imaginary parts are independent zero-mean random Gaussian processes with identical ACF's. Second, the complex waveforms are independent so that the crosscorrelation function between any two waveforms is zero. Therefore, the following results are obtained using a method, that improves Jakes' simulator in order to generate multiple Rayleigh fades, which agree with the theoretical hypothesis above mentioned [12]. As an example, employing $N_{o}=16$ oscillators, we can generate 72 different channel waveforms (needed for a MIMO system with 6 transmitting and receiving antennas) with a crosscorrelation function whose modulus is less than 0.07 .

Now, let us start by examining the numerical results on the impact of spatial and temporal correlations on the capacity. Fig. 2 shows the comparison of the simulated LCR of the capacity for $(4,4)$ and $(6,6)$ systems with that predicted by means of the Gaussian process approximation discussed in section III. The LCR is normalized to the Doppler frequency $f_{D}$, while in the abscissa there is the capacity level $C_{T}$ normalized to the mean $\mathbb{E}[C]$. For this simulation and for what follows the product $f_{D} T$ is equal to 0.02 where $T$ is the burst duration. Note that the analytical model fits very well demonstrating the validity of the Gaussian hypothesis for the capacity, not only for the first-order analysis [9] but also for the process over time.

In Fig. 3 the normalized $\mathrm{ACF} \rho_{c}(\tau)$ of the capacity, for different systems for a SNR of $20 \mathrm{~dB}$ is reported as a function of the normalized time-lag $\tau f_{D}$ and compared to that of the channel $\left(J_{0}\left(2 \pi \tau f_{D}\right)\right)$, where $\tau=k T$ and $k$ is the time index. For the systems considered, the ACF has only a small dependence on the number of antennas, even if a reduction of the amplitude of the oscillations can be observed for higher 


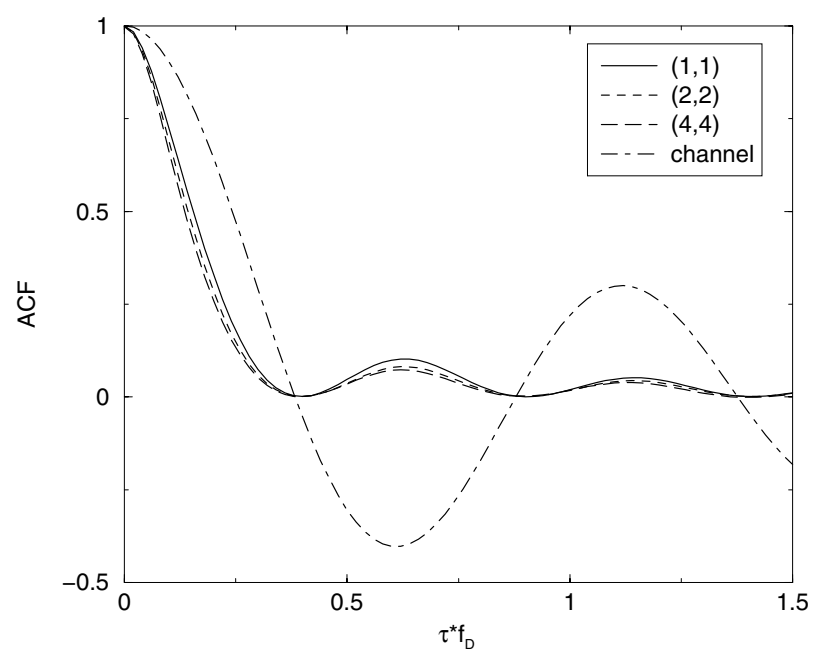

Fig. 3. Normalized ACF of the capacity in spatially uncorrelated channels for $P=20 \mathrm{~dB}$.

order systems. It is interesting to note that when the ACF of the channel cross the zero, the ACF's of the capacities are zero. This is due to the fact that each channel is complex Gaussian: therefore, if we consider a MIMO channel at different instants with a time-lag $\Delta T$ such that the ACF of the channels are zero, the two matrices $\mathbf{H}(t)$ and $\mathbf{H}(t+\Delta T)$ have independent entries, leading to independent capacities.

The normalized (respect to $f_{D}$ ) LCR and the normalized AFD of the capacity for different MIMO systems are reported in Fig. 4 and Fig. 5, respectively. Note that in the abscissa there is the capacity fade probability or outage probability $P_{\text {out }}=\mathbb{P}\left\{C<C_{T}\right\}$. This kind of normalization allow us to better compare the burstiness of different systems at the same outage probability. The AFD curves are easily evaluated taking into consideration formula (11). If we look at low $P_{\text {out }}$, systems with high diversity, i.e. a high $n_{R} / n_{T}$ ratio, have low $L C R$ and high $A F D$. In general, low $A F D$ can be obtained with low numbers of antennas both at the transmitter and at the receiver.

Now, introducing the spatial correlation at transmit and receive antennas as described in section II, we evaluate its impact on the first and second-order statistical properties of the capacity. Here, we consider equally spaced elements at the BTS as well as UE and three different cases of high spatial correlation: at the BTS, at the UE, and both BTS-UE. For the correlation at BTS we have considered three parameters: the average angle of arrival (AOA) $\mu$, the angle spread $\sigma$ and the distance between the transmit antennas $d_{B T S}$. As concerning the correlation at UE, we consider only the antenna spacing $d_{U E}$ and a uniform distribution of the AOA that allow us to use a modified Jakes' simulator for the generation of the channel (remembering that the Jakes' model is based on the assumption of uniforms scatterers on a circle around the UE). In Table I the parameters employed in the following results for the three cases are summarized.

In Fig. 6 we plot the complementary cumulative distribution function (ccdf) $\mathbb{P}\left\{C>C_{T}\right\}$ of the capacity for $(4,4)$ and $(6,6)$ systems, respectively, with and without spatial correlation, compared with the Gaussian approximation to the capacity distribution. As for the Rayleigh and Ricean cases already studied in [9] the Gaussian approximation does remarkably well for both systems considered also in a high spatially correlated scenario corresponding to the case BTS-UE. The strong impact of the spatial correlation is clear, reducing the capacity by around 40\%: a $(6,6)$ system with this spatial correlation is worse than an uncorrelated $(4,4)$.

As concerning the impact of the spatial correlation on the second-order statistics of the capacity, Fig. 7 shows the LCR for a $(4,4)$ system in the three correlated scenarios described above. As a reference, the LCR for $(4,4),(4,1)$ and $(1,4)$ systems without spatial correlation are reported. For a fixed $P_{\text {out }}$, the spatial correlation reduces the LCR and therefore increase the average duration of capacity fades. For the scenario considered the impact of the correlation at BTS seems to be less to that at UE. All the figures given are for a signal-to-noise ratio of $20 \mathrm{~dB}$.

\section{Conclusions}

In this paper we have studied the time-variations of the channel in MIMO systems. The analysis has been carried out in terms of LCR's and AFD's of the (instantaneous) capacity, considering both spatially uncorrelated and spatially correlated environments, where the role of correlation at the base station is kept distinct from that at the mobile terminal. First, it has been shown that a Gaussian first-order approximation is valid even for spatially correlated channels. Then, a Gaussian approximation for the process describing the capacity vs. time has been presented, and validated (for moderate numbers of antennas) by means of simulations. It has been shown that LCR's and AFD's are well approximated by that of a Gaussian process. From a numerical point of view, it has been shown that increasing the number of receiving antenna decreases the level crossing rate, and at the same time increases the average duration of capacity fades, for a fixed outage probability.

TABLE I

SPATIAL CORRELATION PARAMETERS.

\begin{tabular}{c||cccc}
\hline & $\sigma$ & $\mu$ & $d_{B T S}$ & $d_{U E}$ \\
\hline \hline $\mathrm{UE}$ & $20^{\circ}$ & $0^{\circ}$ & $10 \lambda$ & $0.2 \lambda$ \\
\hline $\mathrm{BTS}$ & $5^{\circ}$ & $90^{\circ}$ & $5 \lambda$ & $\lambda$ \\
\hline BTS-UE & $5^{\circ}$ & $90^{\circ}$ & $5 \lambda$ & $0.2 \lambda$ \\
\hline
\end{tabular}




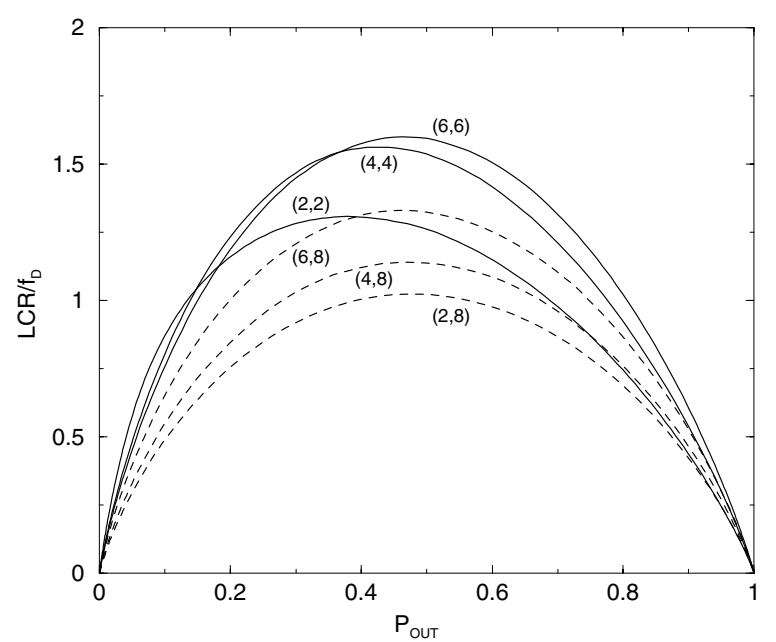

Fig. 4. LCR of the capacity without spatial correlation for different MIMO systems with $P=20 \mathrm{~dB}$. Note that in the abscissa there is the outage probability.

\section{REFERENCES}

[1] J.H. Winters, "On the Capacity of Radio Communication Systems with Diversity in a Radio Fading Environment," IEEE J. on Selected Areas in Comm., vol. 5, no. 5, June 1987.

[2] E. Telatar, "Capacity of Multi-antenna Gaussian Channels," European Trans. on Telecomm., vol. 10, pp. 585-595, Nov.-Dec. 1999.

[3] G.J. Foschini and M.J. Gans, "On Limits of Wireless Communications in a Fading Environment when using Multiple Antennas," Wireless Personal Communications, vol. 6, pp. 311-335, 1998.

[4] IEEE JSAC, special issue on MIMO systems- part I to appear March 2003, part II to appear June 2003.

[5] A. Abdi and M. Kaveh, "A Space-time Correlation Model for Multielement Antenna Systems in Mobile Fading Channels", IEEE J. on Selected Areas in Comm., vol. 20 no. 3, pp. 550-560, April 2002.

[6] 3GPP, A standardised set of MIMO radio propagation channels, TSGR\#23 R1-01-1179, Jeju, Korea, November 19-23, 2001.

[7] Da-Shan Shiu et.al., "Fading Correlation and Its Effect on the Capacity of Multielement Antenna systems," IEEE Trans. on Comm., vol. 48, no. 3, pp 502-512, March 2000.

[8] W. C. Jakes, Microwave Mobile Communications. Piscataway, New Jersey, 08855-1331: IEEE Press, IEEE press classic reissue ed., 1995.

[9] P. J. Smith and M. Shafi, "On a Gaussian Approximation to the Capacity of Wireless MIMO Systems," International Conf. on Comm., ICC 2002, pp. 331-339, IEEE, 2002.

[10] H. Ge, K. D. Wong, M. Barton, and J. C. Liberti, "Statistical Characterization of Multiple-Input Multiple-Output (MIMO) Channel Capacity," Wireless Comm. and Networking Conf., WCNC 2002, IEEE, 2002.

[11] G. L. M.R. Leadbetter and H. Rootzen, Extremes and Related Properties of Random Sequences and Processes. Springer, New York, 1983.

[12] Y. Li and X. Huang, "The Generation of Independent Rayleigh Faders", IEEE International Conf. on Comm., ICC 2000, vol.1, pp. 41-45, 2000.

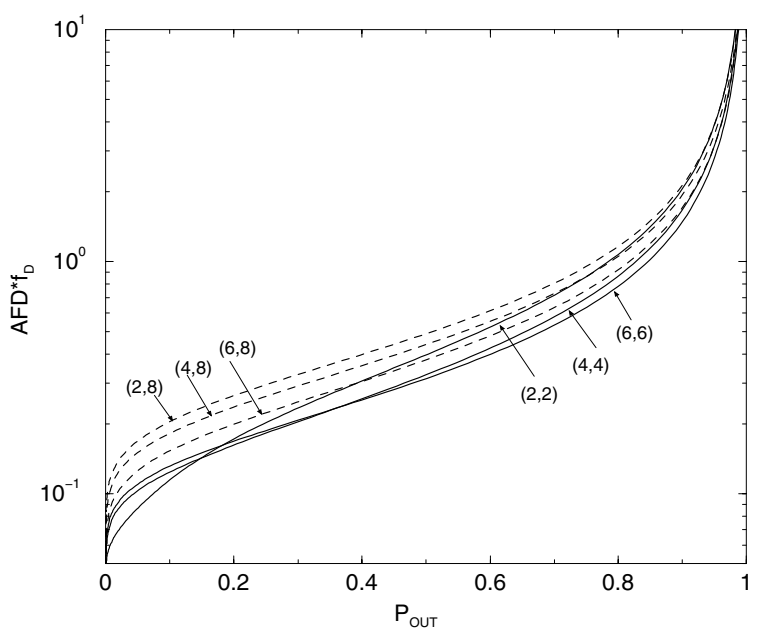

Fig. 5. AFD of the capacity, same systems as in Fig. 4.

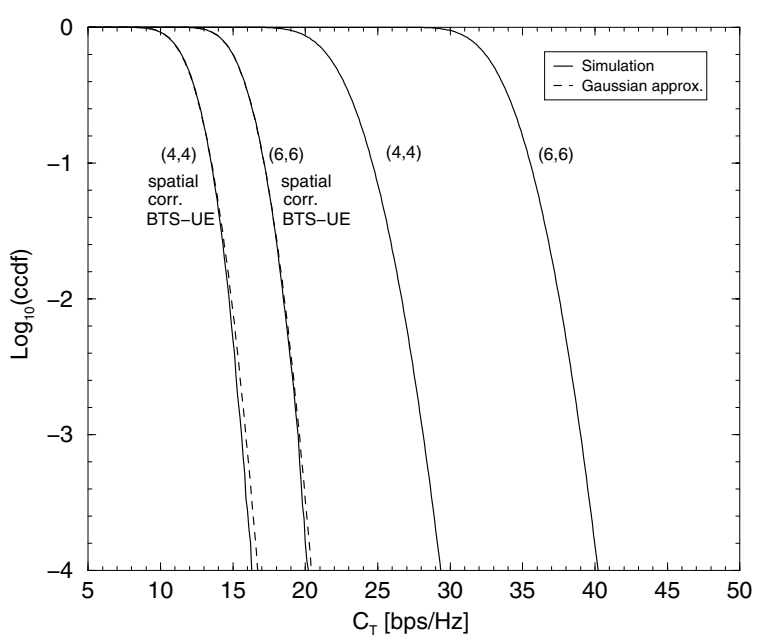

Fig. 6. Complementary cdf (ccdf) of the capacity, $(4,4)$ and $(6,6)$ MIMO for $P=20 \mathrm{~dB}$, with and without spatial correlation (continous line) and the capacity ccdf predicted with the Gaussian assumption.

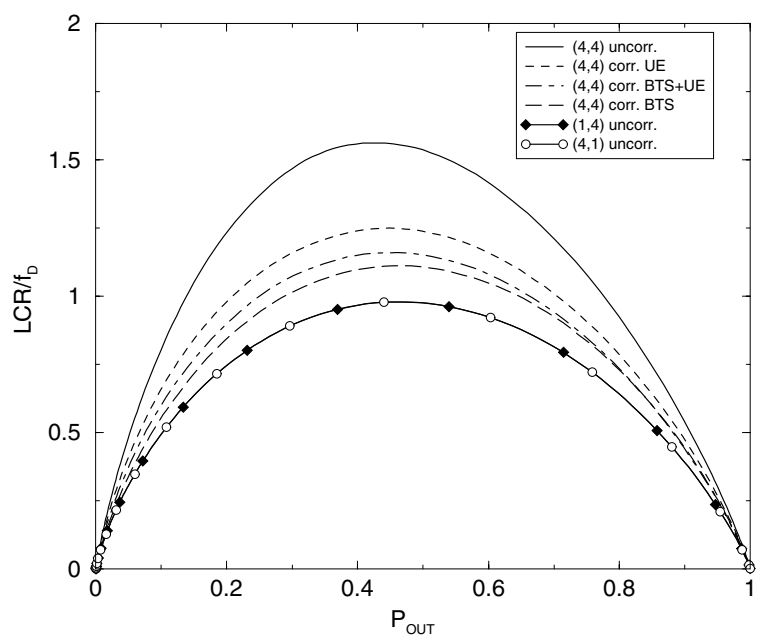

Fig. 7. LCR for different spatial correlation at BTS and UE with $P=20 \mathrm{~dB}$. 